\title{
Multi-component mixture of dipolar hard spheres with surface adhesion*
}

\author{
I.A.Protsykevich \\ Institute for Condensed Matter Physics \\ of the National Academy of Sciences of Ukraine, \\ 1 Svientsitskii Str., 79011 Lviv, Ukraine
}

Received September 8, 2003

Solution of the mean spherical approximation for multi-component model of dipolar hard spheres with surface adhesion is obtained using factorization technique pioneered by Wertheim and Baxter. For the sake of illustration numerical calculations for the dielectric constant of the two-component version of the model are carried out.

Key words: mean spherical approximation, dipolar hard spheres, adhesion, dielectric constant

PACS: 82.70.Dd, 61.20.-p, 61.20.Gy, 61.20.Ne, 61.20.Qg, 02.30.Rz

\section{Introduction}

Mean spherical approximation (MSA) [1] occupies a special place among the liquid state integral equation theories [2-4] due to the availability of the analytical solution of the corresponding Ornstein-Zernike (OZ) equation for a number of simple albeit nontrivial models (see, for example [2-14]).

In the present study we propose the method, which can be used to describe the properties of the fluid of multicomponent dipolar hard spheres with surface adhesion. It is based on the analytical solution of the corresponding version of the MSA with the surface adhesion accounted for following Baxter [15]. In the relevant publications of Blum and coworkers multi-component sticky hard-sphere ion-dipole mixture with orientationally dependent stickiness for dipoles of the same size [16] and one-component hard-sphere system with anisotropic adhesion of arbitrary symmetry and electric multipoles [17] have been studied.

\section{The model}

We consider $M$-component adhesive hard-sphere fluid with the number density of each species $s \rho_{s}=N_{s} / V$, hard-sphere diameter $\sigma_{s}$ and dipolar moment $p_{s}$.

*This paper is dedicated to Professor Myroslav Holovko on the occasion of his 60th birthday. 
In addition to hard-sphere and dipolar interaction there is the so-called "sticky" interaction, which is characterized by the adhesion parameter $\Lambda_{\mathrm{st}}\left(\Omega_{12}\right)$, where $\Omega_{12}=$ $\left(\Omega_{1}, \Omega_{2}\right), \Omega_{1}$ is a set of Euler angles that give the orientation of the molecule 1 .

For the model at hand MSA consists of the following OZ equation and closure conditions

$$
\begin{aligned}
& h_{\mathrm{st}}\left(X_{12}\right)=c_{\mathrm{st}}\left(X_{12}\right)+\sum_{u=1}^{M} \rho_{u} \int \mathrm{d} X_{3} h_{s u}\left(X_{13}\right) c_{u t}\left(X_{32}\right), \\
& h_{\mathrm{st}}\left(X_{12}\right)=-1+\Lambda_{\mathrm{st}}\left(\Omega_{12}\right) \delta\left(r-\sigma_{\mathrm{st}}\right), \quad r_{12}<\sigma_{\mathrm{st}}=\frac{1}{2}\left(\sigma_{s}+\sigma_{t}\right), \\
& c_{\mathrm{st}}\left(X_{12}\right)=-\beta U_{\mathrm{st}}\left(X_{12}\right)=-\beta p_{s} p_{t}\left\{\frac{\mathbf{s}_{1} \mathbf{s}_{2}}{\left|\mathbf{r}_{12}\right|^{3}}-\frac{3 \mathbf{s}_{1} r_{12} \mathbf{r}_{12} \mathbf{s}_{2} \mathbf{r}_{12}}{\left|\mathbf{r}_{12}\right|^{5}}\right\}, \quad r_{12}>\sigma_{\mathrm{st}},
\end{aligned}
$$

where $h_{\mathrm{st}}\left(X_{12}\right)=g_{\mathrm{st}}\left(X_{12}\right)-1$ is the pair correlation function, $g_{\mathrm{st}}\left(X_{12}\right)$ is the pair distribution function, $c_{\mathrm{st}}\left(X_{12}\right)$ is the direct correlation function, $U_{\mathrm{st}}\left(X_{12}\right)$ is the electrostatic pair potential, $r_{12}$ is the distance between the particles, $X_{12}=\left(X_{1}, X_{2}\right)$, $X_{1}=\left(\vec{r}_{1}, \Omega_{1}\right), \beta=1 / k_{b} T, \vec{s}_{1}=\vec{r}_{1} /\left|\vec{r}_{1}\right|$ is the unit vector and $\delta(r)$ is the Dirac delta-function.

Solution of the present MSA problem is based on the Wiener-Hopf factorization technique developed by Wertheim [18] and Baxter [19,20]. In order to consistently account for the orientation dependencies, the technique developed by Blum and co-workers [6-8] is utilized. According to this method all orientation dependent functions are presented in orientational-invariant form

$$
f_{\mathrm{st}}\left(X_{12}\right)=\sum_{\mathrm{mnl}} f_{\mathrm{st}}^{\mathrm{mnl}}\left(r_{12}\right) \Phi_{00}^{\mathrm{mnl}}\left(\Omega_{1}, \Omega_{2}, \Omega_{r_{12}}\right)
$$

where the linear symmetry of the dipoles is accounted for and $\Omega_{r_{12}}$ is a set of Euler angles, which defined the orientation of the vector $\vec{r}_{12}$. This vector connects the centers of masses of the particles 1 and 2 .

$$
\begin{aligned}
\Phi_{00}^{\mathrm{mnl}}\left(\Omega_{1}, \Omega_{2}, \Omega_{r}\right)= & ((2 m+1)(2 n+1))^{1 / 2} \sum_{\mu \nu \lambda}\left(\begin{array}{ccc}
m & n & l \\
\mu & \nu & \lambda
\end{array}\right) \\
& \times D_{0 \mu}^{m}\left(\Omega_{1}\right) D_{0 \nu}^{n}\left(\Omega_{2}\right) D_{0 \lambda}^{l}\left(\Omega_{r}\right), \\
f_{\mathrm{st}}^{\mathrm{mnl}}\left(r_{12}\right)= & (2 l+1)((2 m+1)(2 n+1))^{1 / 2} \sum_{\mu \nu \lambda}\left(\begin{array}{ccc}
m & n & l \\
\mu & \nu & \lambda
\end{array}\right) \\
& \times \int \mathrm{d} \Omega_{1} \mathrm{~d} \Omega_{2} \mathrm{~d} \Omega_{r_{12}} D_{0 \mu}^{* m}\left(\Omega_{1}\right) D_{0 \nu}^{* n}\left(\Omega_{2}\right) D_{0 \lambda}^{* l}\left(\Omega_{r_{12}}\right) f_{\mathrm{st}}\left(X_{12}\right) .
\end{aligned}
$$

Here a standard notation is used for the 3- $j$ Wigner symbols and for generalized spherical functions.

In terms of the rotational invariant expansion, the coefficients closure conditions (2) and (3) are as follows:

$$
h_{\mathrm{st}}^{000}(r)=-1+\Lambda_{\mathrm{st}}^{000} \delta\left(r-\sigma_{\mathrm{st}}^{-}\right), \quad r<\sigma_{\mathrm{st}},
$$




$$
\begin{array}{rr}
h_{\mathrm{st}}^{110}(r)=\Lambda_{\mathrm{st}}^{110} \delta\left(r-\sigma_{\mathrm{st}}^{-}\right), & r<\sigma_{\mathrm{st}}, \\
h_{\mathrm{st}}^{112}(r)=\Lambda_{\mathrm{st}}^{112} \delta\left(r-\sigma_{\mathrm{st}}^{-}\right), & r<\sigma_{\mathrm{st}}, \\
c_{\mathrm{st}}^{000}(r)=0, & r>\sigma_{\mathrm{st}}, \\
c_{\mathrm{st}}^{110}(r)=0, & r>\sigma_{\mathrm{st}}, \\
c_{\mathrm{st}}^{112}(r)=(10 / 3)^{\frac{1}{2}} \beta p_{s} p_{t} r^{-3}, & r>\sigma_{\mathrm{st}} .
\end{array}
$$

Relations (6)-(8) impose dipole symmetry on the adhesion parameter.

In terms of the rotational-invariants coefficients the set of the OZ equations (1) will have the form similar to that presented in [7]

$$
\tilde{H}_{\mathrm{st}, \chi}^{m n}(k)-\tilde{C}_{\mathrm{st}, \chi}^{m n}(k)=\sum_{u=1}^{M} \sum_{l=0}^{1}(-1)^{\chi} \rho_{u} \tilde{H}_{s u, \chi}^{m l}(k) \tilde{C}_{u t, \chi}^{l n}(k),
$$

where

$$
\begin{aligned}
\tilde{F}_{\mathrm{st}, \chi}^{m n}(k) & =2 \int_{0}^{\infty} \mathrm{d} r \cos (k r) \mathcal{F}_{\mathrm{st}}^{\mathrm{mnl}}(r) \\
\mathcal{F}_{\mathrm{st}, \chi}^{m n}(r) & =2 \pi(-1)^{\chi} \sum_{l}\left(\begin{array}{ccc}
m & n & l \\
\chi & -\chi & 0
\end{array}\right) \int_{r}^{\infty} \mathrm{d} t t P_{l}\left(\frac{r}{t}\right) f_{\mathrm{st}}^{\mathrm{mnl}}(t) \\
\tilde{F}_{\mathrm{st}, \chi}^{m n}(k) & \equiv \tilde{H}_{\mathrm{st}, \chi}^{m n}(k) \quad \text { or } \quad \tilde{C}_{\mathrm{st}, \chi}^{m n}(k) \\
\mathcal{F}_{\mathrm{st}, \chi}^{m n}(r) & \equiv J_{\mathrm{st}, \chi}^{m n}(r) \quad \text { or } \quad \tilde{S}_{\mathrm{st}, \chi}^{m n}(r) \\
f_{\mathrm{st}}^{m n}(r) & \equiv h_{\mathrm{st}}^{m n}(r) \quad \text { or } \quad c_{\mathrm{st}}^{m n}(r)
\end{aligned}
$$

for the model at hand the set of equations (12) will be reduced to the set of three independent sets of equations

$$
\begin{aligned}
\tilde{H}_{\mathrm{st}, 0}^{00}(k)-\tilde{C}_{\mathrm{st}, 0}^{00}(k) & =\sum_{u=1}^{M} \rho_{u} \tilde{H}_{s u, 0}^{00}(k) \tilde{C}_{u t, 0}^{00}(k), \\
\tilde{H}_{\mathrm{st}, 0}^{11}(k)-\tilde{C}_{\mathrm{st}, 0}^{11}(k) & =\sum_{u=1}^{M} \rho_{u} \tilde{H}_{s u, 0}^{11}(k) \tilde{C}_{u t, 0}^{11}(k), \\
\tilde{H}_{\mathrm{st}, 1}^{11}(k)-\tilde{C}_{\mathrm{st}, 1}^{11}(k) & =\sum_{u=1}^{M} \rho_{u} \tilde{H}_{s u, 1}^{11}(k) \tilde{C}_{u t, 1}^{11}(k) .
\end{aligned}
$$

Closure conditions for the functions $J_{\mathrm{st}, \chi}^{m n}(r)$ and $S_{\mathrm{st}, \chi}^{m n}(r)$ are as follows:

$$
\begin{aligned}
S_{\mathrm{st}, \chi}^{m n}(r)=0, & m n \chi=000,110,111, \quad r>\sigma_{\mathrm{st}}, \\
J_{\mathrm{st}, \chi}^{m n}(r)=j_{\mathrm{st}, \chi}^{m n, 0}+j_{\mathrm{st}, \chi}^{m n, 2} r^{2}, & r<\sigma_{\mathrm{st}}, \\
j_{\mathrm{st}, 0}^{00,0}=b_{\mathrm{st}, 0}^{00,0}-\pi \sigma_{\mathrm{st}}^{2}, & j_{\mathrm{st}, 0}^{00,2}=\pi \\
j_{\mathrm{st}, 0}^{11,0}=-\frac{1}{3^{1 / 2}} b_{\mathrm{st}, 0}^{110}-\frac{1}{30^{1 / 2}} b_{\mathrm{st}, 2}^{112}, & j_{\mathrm{st}, 0}^{11,2}=\left(\frac{3}{10}\right)^{1 / 2} b_{\mathrm{st}, 2}^{112}, \\
j_{\mathrm{st}, 1}^{11,0}=-\frac{1}{3^{1 / 2}} b_{\mathrm{st}, 0}^{110}+\frac{1}{120^{1 / 2}} b_{\mathrm{st}, 2}^{112}, & j_{\mathrm{st}, 1}^{11,2}=-\left(\frac{3}{40}\right)^{1 / 2} b_{\mathrm{st}, 2}^{112}
\end{aligned}
$$




$$
b_{\mathrm{st}, p}^{\mathrm{mnl}}=2 \pi\left(\sigma_{\mathrm{st}}\right)^{1-p} \Lambda_{\mathrm{st}}^{\mathrm{mnl}}+2 \pi \int_{\sigma_{\mathrm{st}}^{+}}^{\infty} \mathrm{d} r h_{x y}^{\mathrm{mnl}}(r) r^{1-p} .
$$

\section{General solution}

All three sets of equations (14) are of the same form. Omitting the indices we have

$$
\begin{aligned}
\tilde{H}_{\mathrm{st}}(k)-\tilde{C}_{\mathrm{st}}(k) & =\sum_{u=1}^{M} \rho_{u} \tilde{H}_{s u}(k) \tilde{C}_{u t}(k), \\
J_{\mathrm{st}}(r) & =j_{\mathrm{st}}^{0}+j_{\mathrm{st}}^{2} r^{2}, \quad r<\sigma_{\mathrm{st}}, \\
S_{\mathrm{st}}(r) & =0, \quad r>\sigma_{\mathrm{st}},
\end{aligned}
$$

where the function $J_{\text {st }}(r)$ has a jump discontinuity at $r=\sigma_{\text {st }}$ :

$$
\begin{aligned}
J_{\mathrm{st}}\left(\sigma_{\mathrm{st}}^{+}\right)-J_{\mathrm{st}}\left(\sigma_{\mathrm{st}}^{-}\right) & =j_{\mathrm{st}}^{\text {step }}, \\
\tilde{H}_{\mathrm{st}}(k) & =2 \int_{0}^{\infty} \mathrm{d} r \cos (k r) J_{\mathrm{st}}(r), \\
\tilde{C}_{\mathrm{st}}(k) & =2 \int_{0}^{\infty} \mathrm{d} r \cos (k r) S_{\mathrm{st}}(r) .
\end{aligned}
$$

Using matrix notation we have

$$
\left(I+\left[\rho^{1 / 2}\right] \cdot[\tilde{H}(k)] \cdot\left[\rho^{1 / 2}\right]\right) \times\left(I-\left[\rho^{1 / 2}\right] \cdot[\tilde{C}(k)] \cdot\left[\rho^{1 / 2}\right]\right)=I,
$$

where $I$ is the unit matrix, the symbol "." denotes matrix multiplication, square brackets denote matrices of the order $M, \rho_{\mathrm{st}}^{1 / 2}=\delta_{\mathrm{st}} \rho^{1 / 2}, \delta_{\mathrm{st}}$ is the Kronecker delta. Using Wiener-Hopf factorization method we have

$$
\begin{aligned}
I-\left[\rho^{1 / 2}\right] \cdot[\tilde{C}(k)] \cdot\left[\rho^{1 / 2}\right] & =[\tilde{Q}(k)] \cdot[\tilde{Q}(-k)]^{T} \\
{\left[I+\left[\rho^{1 / 2}\right] \cdot[\tilde{H}(k)] \cdot\left[\rho^{1 / 2}\right]\right] \cdot[\tilde{Q}(k)] } & =\left[[\tilde{Q}(-k)]^{T}\right]^{-1}
\end{aligned}
$$

where the upper index $T$ denotes matrix transpose. Wertheim-Baxter factorization correlation functions are of the following form

$$
\begin{aligned}
{[\tilde{Q}(k)]_{\mathrm{st}} } & =\delta_{\mathrm{st}}-\left(\rho_{s} \rho_{t}\right)^{1 / 2} \int_{\lambda_{\mathrm{ts}}}^{\sigma_{\mathrm{st}}} \mathrm{d} r Q_{\mathrm{st}}(r) \mathrm{e}^{\mathrm{i} k r}, \\
Q_{\mathrm{st}}(r) & = \begin{cases}0, & r<\lambda_{\mathrm{ts}}=\left(\sigma_{t}-\sigma_{s}\right) / 2, \\
\left.\left.\left(2 \pi\left(\rho_{s} \rho_{t}\right)^{1 / 2}\right)^{-1} \int_{-\infty}^{\infty} \mathrm{d} k\left(\delta_{\mathrm{st}}-\tilde{Q}_{\mathrm{st}}\right) k\right)\right) \mathrm{e}^{-\mathrm{i} k r}, & \lambda_{\mathrm{ts}}<r<\sigma_{\mathrm{st}}, \\
0, & r>\sigma_{\mathrm{st}} .\end{cases}
\end{aligned}
$$


After the inverse Fourier transform for the equations (23) and (24) we have

$$
\begin{aligned}
S_{\mathrm{st}}(r) & =-Q_{\mathrm{st}}(r)+\sum_{u=1}^{M} \rho_{u} \int_{\left\{\lambda_{u s} ; \lambda_{u t}+r\right\}}^{\left\{\sigma_{s u} ; \sigma_{t u}+r\right\}} \mathrm{d} t Q_{s u}(t) Q_{t u}(t-r), \\
J_{\mathrm{st}}(r) & =Q_{\mathrm{st}}(r)+\sum_{u=1}^{M} \rho_{u} \int_{\lambda_{t u}}^{\sigma_{u t}} \mathrm{~d} t J_{s u}(|r-t|) Q_{u t}(t),
\end{aligned}
$$

where the upper and lower integration limits are represented by the smallest and largest numbers in braces, respectively. Using the closure conditions (17) and taking into account the relation (19) from the equation (27) at $r<\sigma_{\text {st }}$ we have

$$
\begin{aligned}
& Q_{\mathrm{st}}(r)=a_{\mathrm{st}}+\left(r-\sigma_{\mathrm{st}}\right) b_{\mathrm{st}}+1 / 2\left(r-\sigma_{\mathrm{st}}\right)\left(r-\lambda_{\mathrm{ts}}\right) d_{\mathrm{st}}, \\
& a_{\mathrm{st}}=j_{\mathrm{st}}^{\text {step }} \text {, } \\
& b_{\mathrm{st}}=\left(\left[I-\frac{1}{6}\left[\rho_{t} \sigma_{t}^{3} j_{\mathrm{st}}^{2}\right]\right]^{-1} \cdot\left[\sigma_{t} j_{\mathrm{st}}^{2}\right]\right)_{\mathrm{st}},
\end{aligned}
$$

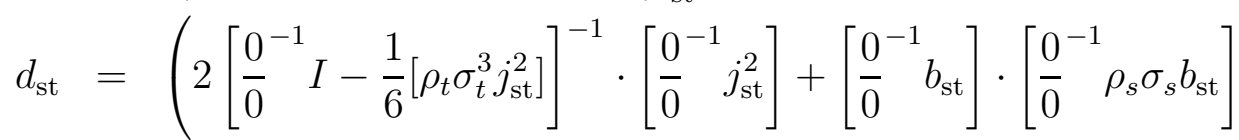

$$
\begin{aligned}
& \left.-2\left[\begin{array}{ll}
\frac{1}{6}^{-1} & b_{\mathrm{st}}
\end{array}\right] \cdot\left[\left[\frac{1}{6}^{-1}\right]^{-1} \rho_{s} a_{\mathrm{st}}\right]\right)_{\mathrm{st}} .
\end{aligned}
$$

\section{Wertheim-Baxter factorization correlation function}

Using the results obtained in the previous section and taking into account the rotationally-invariant indices $m, n$ and $\chi$ Wertheim-Baxter factorization correlation functions can be written as follows:

$$
\begin{aligned}
Q_{\mathrm{st}, \chi}^{m n}(r) & =a_{\mathrm{st}, \chi}^{m n}+\left(r-\sigma_{\mathrm{st}}\right) b_{\mathrm{st}, \chi}^{m n}+1 / 2\left(r-\sigma_{\mathrm{st}}\right)\left(r-\lambda_{\mathrm{ts}}\right) d_{\mathrm{st}, \chi}^{m n}, \\
a_{\mathrm{st}, 0}^{00} & =2 \pi \sigma_{\mathrm{st}} \Lambda_{\mathrm{st}}^{000} \\
b_{\mathrm{st}, 0} & =\frac{\pi}{\Delta} \sigma_{t} \\
d_{\mathrm{st}, 0} & =\frac{2 \pi}{\Delta}+\frac{\pi^{2}}{\Delta^{2}} \zeta_{2} \sigma_{t}-\frac{2 \pi}{\Delta} \sum_{u=1}^{M} \rho_{u} \sigma_{u} a_{u t, 0}, \\
a_{\mathrm{st}, 0}^{11} & =-\frac{2 \pi}{3^{1 / 2}} \sigma_{\mathrm{st}} \Lambda_{\mathrm{st}}^{110}+\frac{4 \pi}{30^{1 / 2}} \sigma_{\mathrm{st}} \Lambda_{\mathrm{st}}^{112} \\
a_{\mathrm{st}, 1}^{11} & =-\frac{2 \pi}{3^{1 / 2}} \sigma_{\mathrm{st}} \Lambda_{\mathrm{st}}^{110}-\frac{2 \pi}{30^{1 / 2}} \sigma_{\mathrm{st}} \Lambda_{\mathrm{st}}^{112}
\end{aligned}
$$

where $\Delta=1-\pi / 6 \zeta_{3} ; \zeta_{p}=\sum_{u=1}^{M} \rho_{u}\left(\sigma_{u}\right)^{p}$.

Coefficients of the Wertheim-Baxter factorization function $b_{\mathrm{st}, 0}^{11}, d_{\mathrm{st}, 0}^{11}, b_{\mathrm{st}, 1}^{11}$ and $d_{\mathrm{st}, 1}^{11}$ follow from the solution of the set of equations

$$
b_{\mathrm{st}, 0}^{11}=\frac{1}{120^{1 / 2}} \sum_{u=1}^{M} \rho_{u} \sigma_{u}^{3} b_{s u, 2}^{112} b_{u t, 0}^{11}+0.3^{1 / 2} \sigma_{t} b_{\mathrm{st}, 2}^{112},
$$




$$
\begin{aligned}
d_{\mathrm{st}, 0}^{11} & =\frac{2}{\sigma_{t}} b_{\mathrm{st}, 0}^{11}+\sum_{u=1}^{M} \rho_{u} \sigma_{u} b_{s u, 0}^{11} b_{u t, 0}^{11}-2 \sum_{u=1}^{M} \rho_{u} b_{s u, 0}^{11} a_{u t, 0}^{11}, \\
b_{\mathrm{st}, 1}^{11} & =-\frac{1}{480^{1 / 2}} \sum_{u=1}^{M} \rho_{u} \sigma_{u}^{3} b_{s u, 2}^{112} b_{u t, 1}^{11}+0.075^{1 / 2} \sigma_{t} b_{\mathrm{st}, 2}^{112}, \\
d_{\mathrm{st}, 1}^{11} & =\frac{2}{\sigma_{t}} b_{\mathrm{st}, 1}^{11}+\sum_{u=1}^{M} \rho_{u} \sigma_{u} b_{s u, 1}^{11} b_{u t, 1}^{11}-2 \sum_{u=1}^{M} \rho_{u} b_{s u, 1}^{11} a_{u t, 1}^{11} .
\end{aligned}
$$

This set of equations have to be supplemented by the additional equation for the parameter $b_{\mathrm{st}, 2}^{112}$. This equation can be obtained from the closure conditions for $c_{\mathrm{st}}^{112}(r)$ at $r>\sigma_{\mathrm{st}}$

$$
\frac{4 \pi}{3} \beta p_{s} p_{t}=-\tilde{C}_{\mathrm{st}, 0}^{11}(k=0)+\tilde{C}_{\mathrm{st}, 1}^{11}(k=0) .
$$

Introducing the quantities

$$
K_{\mathrm{st}}^{m n, p}=\int_{\lambda_{\mathrm{ts}}}^{\sigma_{\mathrm{st}}} \mathrm{d} r r^{p} Q_{\mathrm{st}}^{m n}(r),
$$

equation (31) can be written as follows

$$
\begin{aligned}
\frac{4 \pi}{3} \beta p_{s} p_{t}= & -K_{\mathrm{st}, 0}^{11,0}-K_{t s, 0}^{11,0}+\sum_{u=1}^{M} \rho_{u} K_{s u, 0}^{11,0} K_{t u, 0}^{11,0} \\
& +K_{\mathrm{st}, 1}^{11,0}+K_{t s, 1}^{11,0}-\sum_{u=1}^{M} \rho_{u} K_{s u, 1}^{11,0} K_{t u, 1}^{11,0}
\end{aligned}
$$

where

$$
K_{\mathrm{st}, \chi}^{11,0}=\sigma_{s} a_{\mathrm{st}, \chi}^{11}-\frac{1}{2} \sigma_{s}^{2} b_{\mathrm{st}, \chi}^{11}-\frac{1}{3} \sigma_{s}^{3} d_{\mathrm{st}, \chi}^{11}
$$

Thus, we obtain a closed set of equations (30) and (33) for the coefficients of the Wertheim-Baxter factorization correlation function and for the dipole-dipole interaction parameter $b_{\mathrm{st}, 2}^{112}$.

\section{Dielectric constant. Numerical calculations}

The expression for the dielectric constant of the dipolar mixture $\epsilon$ was obtained in $[7,21]$. For the model at hand we have

$$
\frac{\varepsilon-1}{\varepsilon+2}=\operatorname{Tr}\left(\left[\left[q_{1}\right] \cdot\left[q_{2}\right]^{-1}-I\right] \cdot\left[\left[q_{1}\right] \cdot\left[q_{2}\right]^{-1}+2 I\right]^{-1}\right)
$$

where

$$
\begin{aligned}
& {\left[q_{1}\right]=\left(I-\left[\rho^{1 / 2}\right] \cdot\left[K_{\mathrm{st}, 0}^{11,0}\right] \cdot\left[\rho^{1 / 2}\right]\right) \times\left(I-\left[\rho^{1 / 2}\right] \cdot\left[K_{\mathrm{st}, 0}^{11,0}\right] \cdot\left[\rho^{1 / 2}\right]\right)^{T},} \\
& {\left[q_{2}\right]=\left(I-\left[\rho^{1 / 2}\right] \cdot\left[K_{\mathrm{st}, 1}^{11,0}\right] \cdot\left[\rho^{1 / 2}\right]\right) \times\left(I-\left[\rho^{1 / 2}\right] \cdot\left[K_{\mathrm{st}, 1}^{11,0}\right] \cdot\left[\rho^{1 / 2}\right]\right)^{T} .}
\end{aligned}
$$


Table 1. Dielectric properties of the two-component dipolar hard-sphere fluid. Hard spheres of species 1 has a diameter $\sigma_{1}=1$ and hard-spheres size of species 2 is $\sigma_{2}=2^{1 / 3} \sigma_{1}$. The reduced total density is $\rho^{*}=\rho_{1} \sigma_{1}^{3}+\rho_{2} \sigma_{2}^{3}=0.8$. The dipole moment of species 1 and 2 are such that when $\rho_{2}=0$ (pure fluid 1) then $y=2.5$ and when $\rho_{1}=0$ (pure fluid 2) then $y=1.5$, where $y=4 \pi / 9 \beta \sum_{s} \rho_{s} p_{s}^{2}$. Here $\Lambda_{\mathrm{st}}^{\mathrm{mnl}}$ is treated as a fitting parameter.

\begin{tabular}{|c|c|c|c|c|}
\hline$X_{1}=\rho_{1} / \rho$ & $y$ & $\begin{array}{c}E_{\mathrm{MSA}} \\
{[6]}\end{array}$ & $\begin{array}{c}E_{\mathrm{MC}} \\
{[6]}\end{array}$ & $\begin{array}{c}\Lambda_{\mathrm{st}}^{110}=0, \quad \Lambda_{11}^{112}=-0.14, \\
\Lambda_{12}^{112}=-0.18, \quad \Lambda_{22}^{112}=-0.24\end{array}$ \\
\hline 0.0 & 1.5 & 11.5 & 13.65 & 13.66 \\
0.3 & 1.7 & 13.4 & 17.4 & 17.5 \\
0.8 & 2.2 & 19.1 & 26.0 & 26.2 \\
1.0 & 2.5 & 23.5 & 37.2 & 36.9 \\
\hline
\end{tabular}

To calculate the dielectric constant of the system, the solution of the set of equations (30) and (33) has to be obtained. This is a set of highly nonlinear equations, which can be solved only using the numerical methods, for example using the Newton's method. To use this method it is important to have a sufficiently accurate initial guess for the unknowns of the problem. In our study we start from the twocomponent version of the model with hard spheres of the same size and dipolar moment. For such a model, the dipole-dipole interaction parameter $b_{\mathrm{st}, 2}^{112}$ is the same for both species and can be obtained from the corresponding nonlinear equation. All the rest of the unknown coefficients can be easily obtained from $b_{\mathrm{st}, 2}^{112}$. Gradually changing the ratio of the hard-sphere sizes and dipolar moments one can get solution of the set of equations (30) and (33) for the two-component system with arbitrary hard-sphere sizes and dipolar moments.

For the sake of illustration in table 1 we present the dependance of the dielectric constant of the two-component mixture on the concentration of the first species $X_{1}=\rho_{1} / \xi_{0}$ with the value of the adhesion constant $\Lambda_{\mathrm{st}}^{\mathrm{mnl}}$ chosen so as to fit the corresponding $\mathrm{MC}$ values. Good agreement between $\mathrm{MC}$ and theoretical predictions in the whole range of concentrations with only one value for $\Lambda_{\mathrm{st}}^{\mathrm{mnl}}$ shows that the model at hand can be used to correlate the experimental data with the dielectric properties of multi-component polar fluids.

\section{References}

1. Lebowitz J.L., Percus J.K. // Phys. Rev., 1966, vol. 144, p. 251.

2. Yukhnovski I.R., Holovko M.F. Statistical Theory of Classical Equilibrium Systems. Kyiv, Naukova Dumka, 1980.

3. Hansen J.-P., McDonald I.R. Theory of Simple Liquids. 2nd edition. New York, Academic, 1986. 
4. Gray C.G., Gubbins K. Theory of Molecular Fluids. Vol. 1: Fundamentals. Oxford, Clarendon Press, 1984.

5. Kalyuzhnyi Y.V., Cummings P.T. Equations of State for Fluids and Fluid Mixtures. Elsevier, 2000.

6. Blum L., Toruella A.J. // J. Chem. Phys., 1972, vol. 56, No. 6, p. 303-310.

7. Blum L. // J. Chem. Phys., 1972, vol. 57, No. 5, p. 1862-1869.

8. Blum L. // J. Chem. Phys., 1973, vol. 58, No. 8, p. 3295-3303.

9. Blum L. // J. Stat. Phys., 1978, vol. 18, No. 5, p. 451-474.

10. Blum L., Wei D.Q. // J. Chem. Phys., 1987, vol. 87, No. 1, p. 555-565.

11. Wei D., Blum L. // J. Chem. Phys., 1987, vol. 87, No. 5, p. 2999-3007.

12. Golovko M.F., Protsykevich I.A. // Chem. Phys. Lett., 1987, vol. 142, No. 6, p. 463469.

13. Golovko M.F., Protsykevich I.A. // J. Stat. Phys., 1989, vol. 54, No. 3/4, p. 707-733.

14. Pizio O.O., Holovko M.F., Trokhymchuk A.D. // Acta Chemica Hungarica, 1988, vol. 125 , p. $385-402$.

15. Baxter R.J. // J. Chem. Phys., 1968, vol. 49, p. 2770.

16. Wei D., Blum L. // J. Chem. Phys., 1988, vol. 89, p. 1091-1101.

17. Blum L., Cummings P.T., Bratko D. // J. Chem. Phys., 1990, vol. 92, p. 3741-3747.

18. Wertheim M.S. // Phys. Rev. Lett., 1963, vol. 10, p. 321.

19. Baxter R.J. // Austral. J. Phys., 1968, vol. 21, No. 5, p. 563-569.

20. Baxter R.J. // J. Chem. Phys., 1970, No. 9.

21. Cummings P.T., Blum L. // J. Chem. Phys., 1986, vol. 85, No. 11, p. 6658-6667.

\section{Багатокомпонентна суміш дипольних твердих сфер з поверхневим прилипанням}

\section{І.А.Процикевич}

Інститут фізики конденсованих систем НАН України, 79011 Львів, вул. Свєнціцького, 1

Отримано 8 вересня 2003 р.

На основі методу факторизації, започаткованого Вертхаймом та Бакстером, отриманий аналітичний розв'язок середньосферичного наближення для багатокомпонентної моделі дипольних твердих сфер з поверхневою липкістю. 3 метою ілюстрації виконані чисельні розрахунки діелектричної сталої двокомпонентного варіанту моделі.

Ключові слова: середньосферичне наближення, дипольні тверді сфери, адгезія, діелектрична постійна

PACS: 82.70.Dd, 61.20.-p, 61.20.Gy, 61.20.Ne, 61.20.Qg, 02.30.Rz 